\title{
Arrhythmia by ECG Finding
}

National Cancer Institute

\section{Source}

National Cancer Institute. Arrhythmia by ECG Finding. NCI Thesaurus. Code $C 111075$.

An arrhythmia that is detected on an electrocardiograph. 